\title{
Research Paper \\ Relationship of Perceived Social Support With Health-Promoting Lifestyle in Women Participating in National Breast Cancer Early Detection Program
}

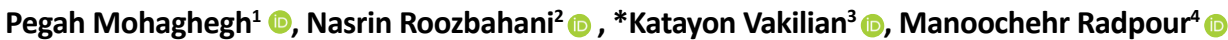

1. Department of Community Medicine, School of Medicine, Arak University of Medical Sciences, Arak, Iran

2. Department of Health, School of Health, Arak University of Medical sciences, Arak, Iran.

3. Department of Midwifery, School of Medicine, Arak University of Medical Sciences, Arak, Iran

4. Vice Chancellor for Health, Arak University of Medical sciences, Arak, Iran.

\begin{tabular}{|c|c|}
\hline $\begin{array}{l}\text { Use your device to scan } \\
\text { and read the article online }\end{array}$ & iftation Mohaghegh P, Roozbahani N, Vakilian K, Radpour M. [Relationship of Perceived Social Supp \\
\hline 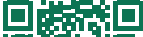 & Promoting Lifestyle in Women Participating in National Breast Cancer Early Detection Program (Persian)]. Iranian Journal of \\
\hline H.t. & Psychiatry and Clinical Psychology. 2021; 26(4):464-477. http://dx.doi.org/10.32598/ijpcp.26.4.3311.1 \\
\hline 19t2y & doil'http://dx.doi.org/10.32598/ijpcp.26.4.3311.1 \\
\hline
\end{tabular}

Received: 19 Jan 2020

Accepted: 08 Jun 2020

Available Online: 01 Jan 202

Key words:

Health-promoting lifestyle, Perceived social support, Breast cancer early detection

\begin{abstract}
Objectives Due to the importance of social support and healthy lifestyle, this study aims to evaluate the relationship of perceived social support with health-promoting lifestyle in women who participated in national breast cancer early detection program.

Methods In this cross-sectional study, 1000 women participating in breast cancer early detection program in Arak, Iran were selected from 16 health centers using a convenience sampling method. Two questionnaires of Perceived Social Support (PSS) and Health-Promoting Lifestyle Profile (HPLP) were completed on behalf of them. To describe the variables, mean, standard deviation, frequency and percentage were used. The Pearson correlation test was used for examining the correlation between study variables. Data analysis was conducted in SPSS V. 23 software.

Results The Mean \pm SD age of women was $33.78 \pm 11.30$ years. There was a significant correlation between perceived social support and health-promoting lifestyle $(r=0.42)$. Regarding HPLP dimensions, perceived social support had the highest and lowest correlation with spiritual growth $(r=0.46)$ and physical activity (0.26) dimensions, respectively. Regarding the PSS dimensions, health-promoting lifestyle had the highest and lowest correlation with family support $(r=0.40)$ and the support from friends $(r=0.30)$, respectively. Conclusion There is a direct and significant relationship between perceived social support and healthpromoting lifestyle in women. Therefore, by increasing their social support, it is possible to promote their healthy lifestyle.
\end{abstract}

\section{Extended Abstract}

\section{Introduction}

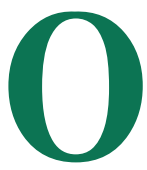

ne of the determinants the community health is social support which indicates the importance of the social dimension of human beings, and has received increasing attention in recent years. Social support refers to the love, dignity, consolation, help, and facilities that others provide to a person. Social support increases a person's adaptability, satisfaction, and efficiency in life $[1,2]$. Perceived social support is a mental feeling about belonging, accepting, and receiving help from others when needed [3]. Social support sources may include family, friends, relatives, social organizations, or even those available at workplace and educational settings. Since more than $70 \%$ of diseases are related to people's lifestyles [9], increasing the awareness

* Corresponding Author:

Katayon Vakilian, PhD.

Address: Department of Midwifery, School of Medicine, Arak University of Medical Sciences, Arak, Iran.

Tel: +98 (86) 34173405

E-mail: dr.kvakilian@arakmu.ac.ir 
of the influence of perceptual social support on healthpromoting behaviors can be effective in disease prevention [9]. A healthy lifestyle includes all the actions and behaviors that reduce exposure to disease in people. These behaviors include proper diet, sleep and activity, exercise and weight control, no smoking, no alcohol consumption, no drug abuse, and immunization. Health-promoting lifestyle is measured by the Health-Promoting Lifestyle Profile (HPLP) questionnaire. It has six dimensions of nutrition, physical activity, health responsibility, stress management, spiritual growth, and interpersonal relationships [13]. Haji Amiri et al. showed that the increase of social support improves the health-promoting lifestyle of postpartum women [14]. Few studies have focused on the interaction effect of social support on women's health-promoting behaviors including their participation in early diagnosis and screening programs. With the implementation of the national plan for early breast cancer screening in Arak, Iran, we found an opportunity to conduct this study on women participated in this screening program to assess their perceived social support and healthpromoting lifestyle, and the relationship between them

\section{Methods}

In this cross-sectional study, 1000 women (aged 15-71 years) participating in the breast cancer screening program were selected using a convenience sampling technique from 16 health centers in Arak, Iran (about 62 from each center). The questionnaires were completed after providing explanations to the participants about the study objectives and obtaining their informed consent. The 12-item Perceived Social Support (PSS) questionnaire was used to evaluate their perceived social support [26]. This questionnaire measures social support from three sources: family, friends, and significant others. The 49-item HPLP was used to assess their health-promoting behaviors [13]. The mean, standard deviation, frequency, and percentage were used to describe the collected data, and the Pearson correlation test was used to measure the relationship between the variables.

\section{Results}

Of 1000 women, $77.6 \%$ were married, $74.4 \%$ were housewives, $85.5 \%$ were living in urban areas, $10.5 \%$ were

Table 1. Correlation of health-promoting lifestyle with perceived social support and demographic factors in women

\begin{tabular}{|c|c|c|c|c|c|c|}
\hline \multirow{2}{*}{ Variable } & \multirow{2}{*}{$\mathbf{N}$} & \multirow{2}{*}{ Mean $\pm S D$} & \multicolumn{2}{|c|}{ Perceived Social Support } & \multicolumn{2}{|c|}{ Health-promoting Lifestyle } \\
\hline & & & $\mathbf{r}$ & Sig. & $\mathbf{r}$ & Sig. \\
\hline Health-promoting lifestyle & 883 & $25.35 \pm 117.45$ & 0.429 & 0.000 & 1 & 0.000 \\
\hline Perceived social support & 1000 & $8.52 \pm 44.82$ & 1 & 0.000 & 0.429 & 0.000 \\
\hline Support from family & 985 & $3.22 \pm 16.07$ & 0.856 & 0.000 & 0.405 & 0.000 \\
\hline Support from friends & 985 & $3.67 \pm 13.68$ & 0.827 & 0.000 & 0.300 & 0.000 \\
\hline Support from significant others & 985 & $3.24 \pm 15.06$ & 0.843 & 0.000 & 0.376 & 0.000 \\
\hline Physical activity & 973 & $4.99 \pm 14.67$ & 0.268 & 0.000 & 0.784 & 0.000 \\
\hline Nutrition & 970 & $4.00 \pm 17.34$ & 0.293 & 0.000 & 0.742 & 0.000 \\
\hline Health responsibility & 936 & $7.34 \pm 31.78$ & 0.355 & 0.000 & 0.905 & 0.000 \\
\hline Spiritual growth & 1000 & $6.48 \pm 24.57$ & 0.462 & 0.000 & 0.899 & 0.000 \\
\hline Stress management & 966 & $2.98 \pm 11.87$ & 0.281 & 0.000 & 0.815 & 0.000 \\
\hline Interpersonal relationships & 962 & $4.31 \pm 17.70$ & 0.418 & 0.000 & 0.857 & 0.000 \\
\hline Age (year) & 984 & $11.30 \pm 33.78$ & -0.110 & 0.001 & -0.136 & 0.000 \\
\hline Educational level & 952 & $4.19 \pm 11.31$ & 0.192 & 0.000 & 0.221 & 0.000 \\
\hline Per capita income (Rials) & 530 & $435170.8 \pm 628142.8$ & 0.136 & 0.002 & 0.144 & 0.002 \\
\hline Body mass index $\left(\mathrm{Kg} / \mathrm{m}^{2}\right)$ & 957 & $4.38 \pm 25.21$ & -0.032 & 0.325 & -0.035 & 0.309 \\
\hline
\end{tabular}


living in suburban areas, and 4\% were rural dwellers. Most of them $(92.1 \%)$ had been covered by different types of health insurance. Statistics reported that $93.1 \%$ had proper social support. Their mean HPLP score was $25.35 \pm 117.45$. Regarding its dimensions, the highest score belonged to health responsibility $(7.34 \pm 31.78)$, while the lowest score belonged to stress management $(2.98 \pm 11.87)$. On the other hand, their mean PSS score was reported $8.52 \pm 44.82$. Regarding its dimensions, the highest score belonged to family support (3.22 \pm 16.07$)$, and the lowest score was related to the support from friends $(3.67 \pm 13.68)$. According to the results in Table 1, there was a positive significant correlation between perceived social support and health-promoting lifestyle and their dimensions.

\section{Discussion}

The results of the current study revealed that women with higher social support had a healthier lifestyle. Demographic factors including age, education and income level had a weak significant relationship with their health-promoting lifestyle and perceived social support. Perceived social support had the highest relationship with spiritual growth dimension of HPLP followed by health responsibility, interpersonal relationships, stress management, physical activity, and nutrition dimensions. A higher level of healthpromoting lifestyle can increase social adjustment and life expectancy [19]. The results revealed that the most common perceived social support was family support followed by the support from significant others, and friends. Other studies have also shown a significant relationship between the support from friends as a source of social support and the health-promoting lifestyle in the disabled people [25].

The results of our study showed a positive significant relationship between perceived social support and stress management dimension of HPLP. Bakhshani et al. reported that perceived social support play a moderating role in the incidence or worsening of depression, and is significantly lower in depressed individuals than in non-depressed ones [30]. A study by Abbey et al. on infertile couples reported the effect of spouse support on reducing stress and improving life quality [36]. Their results are consistent with our results regarding the effect of family support on stress reduction. Therefore, by improving social support, it is possible to have a healthy lifestyle and health-promoting behaviors, and consequently prevent and treat many chronic physical and mental diseases.

\section{Ethical Considerations}

\section{Compliance with ethical guidelines}

This study was extracted from a research proposal approved by Arak University of Medical Sciences with an Ethical approval Code: IR.ARAKMU.REC.1397.199. All ethical principles were observed in this study. The participants were informed about the study objectives, and were assured of the confidentiality of their information.

\section{Funding}

The study received financial support the Deputy for Research and Technology of Arak University of Medical Sciences.

\section{Authors contributions}

Conceptualization and methodology: Pegah Mohaghegh, Katayon Vakilian, Nasrin Roozbahani; Editing \& review: Pegah Mohaghegh; Writing: Pegah Mohaghegh and Manoochehr Radpour; Data analysis: Nasrin Roozbahani, Manoochehr Radpour and Pegah Mohaghegh.

\section{Conflicts of interest}

The authors declare no conflict of interest.

\section{Acknowledgements}

The authors are grateful to the Research Deputy, Arak University of Medical Sciences, Arak, Iran. 


\section{بررسى ارتباط حمايت اجتماعى ادراكشده با سبك زندكى ارتقادهنده سلامت در زنان شركت كثنده

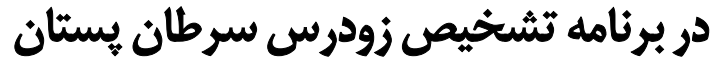

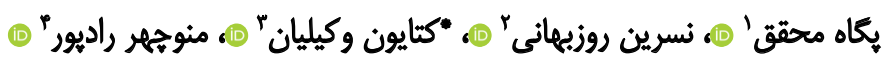

1. كروه هزشكى اجتماعى، دانشكده يزشكى، دانشعاه علوميزشكى اراك، اراك، ايران.

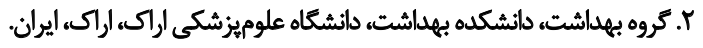

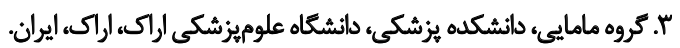

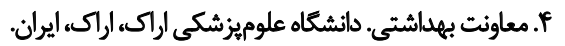

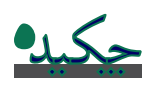

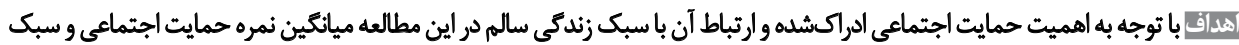

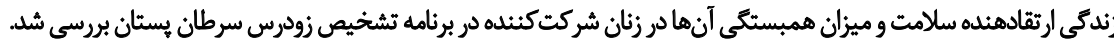

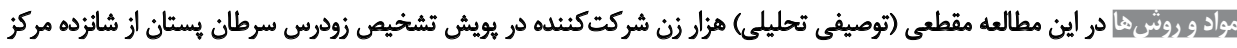

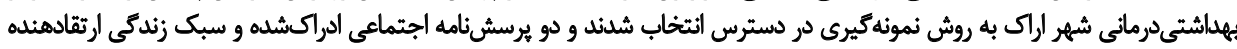

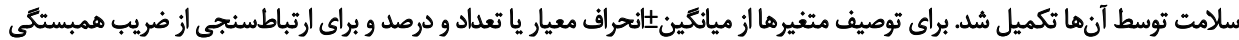

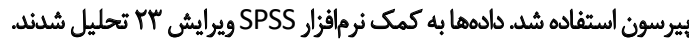

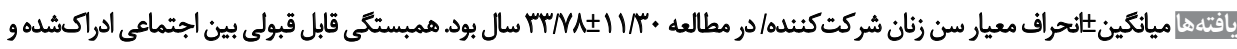

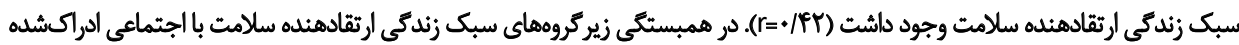

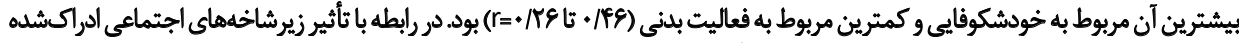

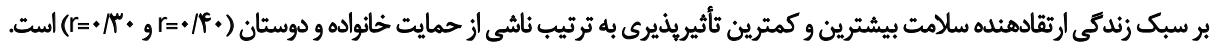

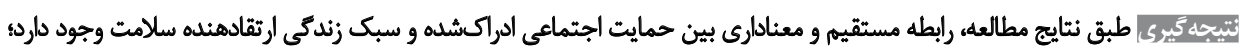

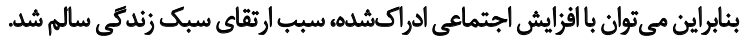

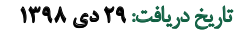
تاريخ يذيرش: 19 اخرداد

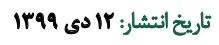

به دريافت آن كردهاند و حمايت اجتماعي ذهني و يا دركشده

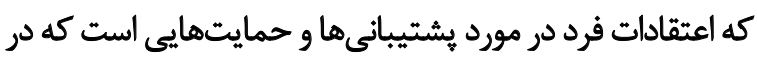

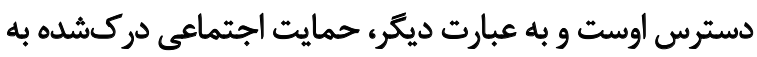

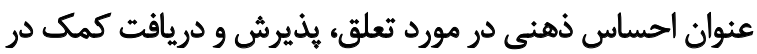

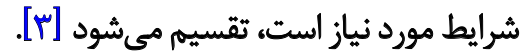

حمايت اجتماعي با مزاياي متعدد رواني، از جمله بهبود اعتماد

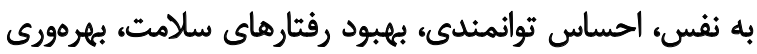

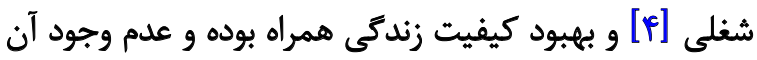
با تظاهرات روانى و سطح سلامت هايينتر همراه است. مطالعات

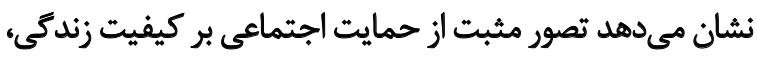

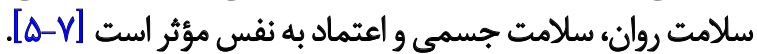

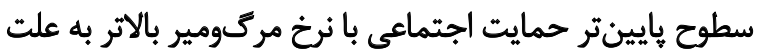

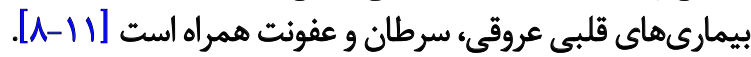

dâa

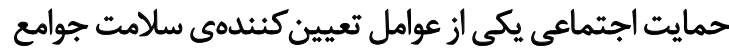

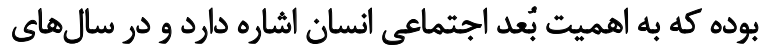

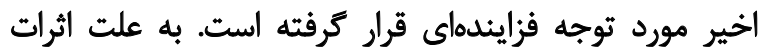

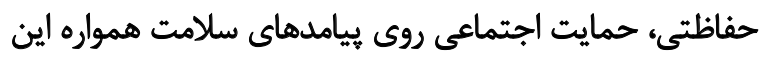

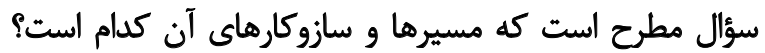

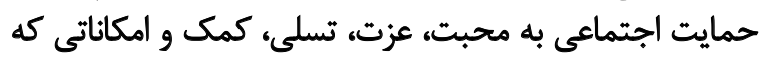

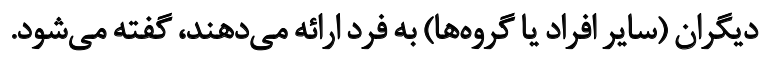

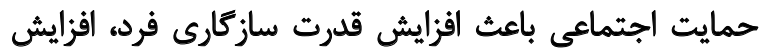

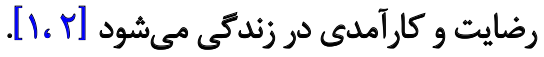
به طور كلى حمايت اجتماعى به دو دسته حمايت اجتماعى

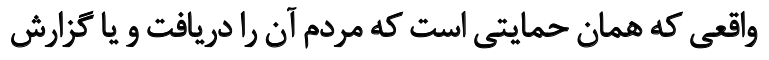


[بr]. بين متغيرهاى خودكارآهدى، سطح تحصيلات، ميزان

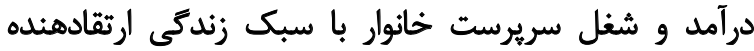
سلامت رابطه معنادارى وجود دارد [بr.

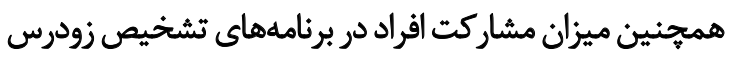

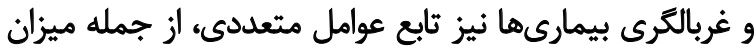

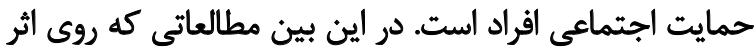

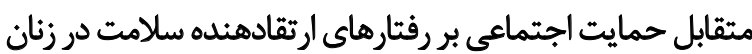

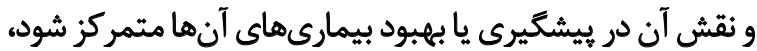

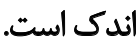
با اجرايى شدن طرح ملى يويش تشخيص زودرس سرطان

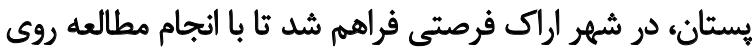

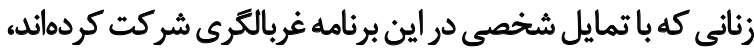

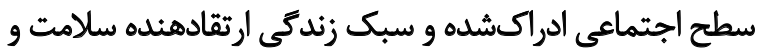
ارثباط آنها با يكديكر بررسى شود.

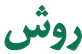

در اين مطالعه مقطعى هزار نفر از زنان (1- (1-اساله)

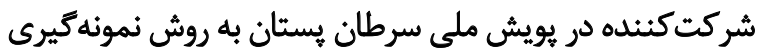

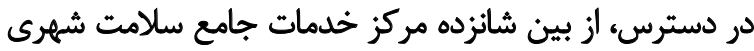

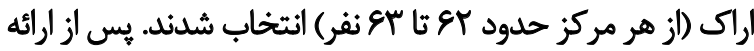

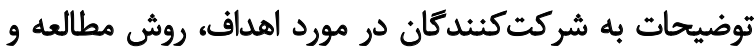

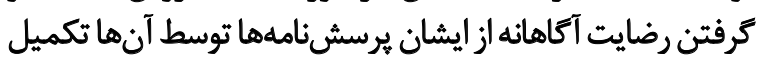

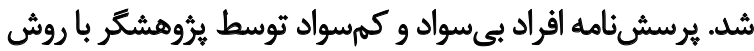

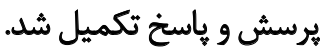

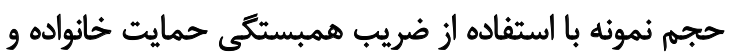

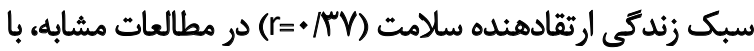

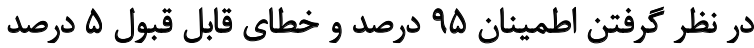

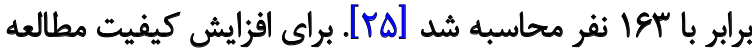

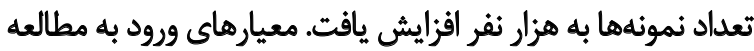

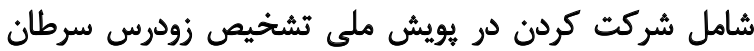

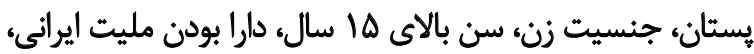
سكونت در شهرستان اراك و تكميل فرم رضايت آكاهاهن بانه بوده است.

براى بررسى حمايت اجتماعى از يرسشىنامه دوازدهسؤالى إلى

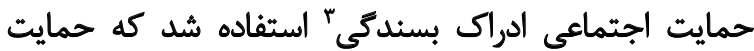

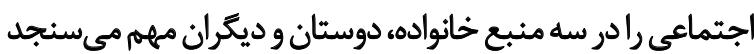

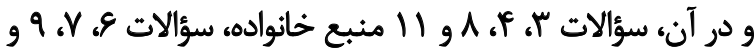

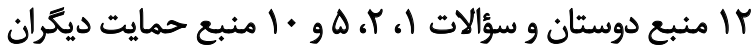

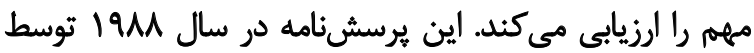

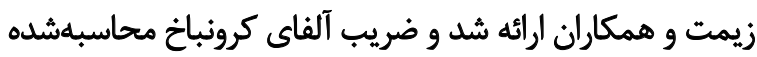

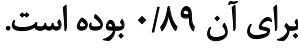

3. Perceived Social Support Questionnaire

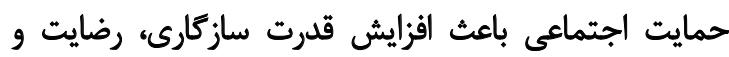

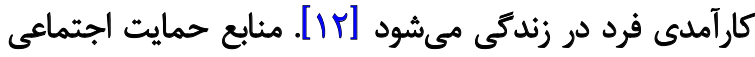

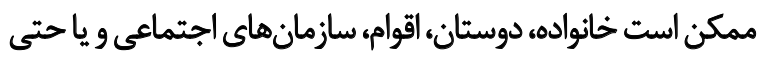

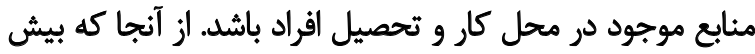

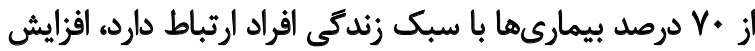

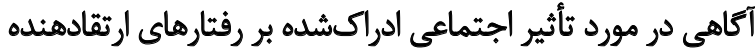

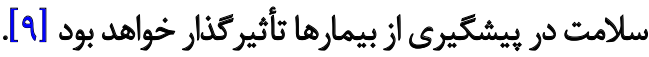
سبك زندگى سالم شامل تمام اقدامات و فعاليتهاو رفتارهايى

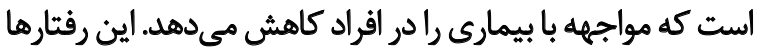

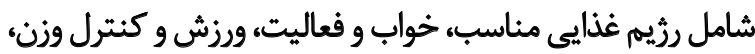

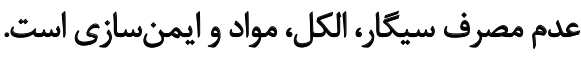

سبك زندگى ارتقادهنده سلامت مى تواند توسط بروفايل سبك

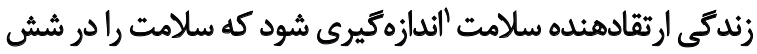

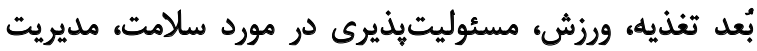

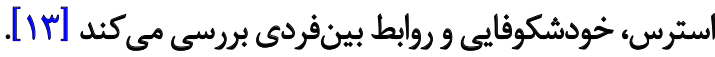
طبق نتايج مطالعه حاجى اميرى و همكاران افزايش حمايت

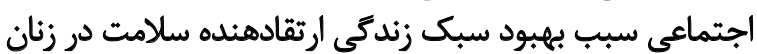

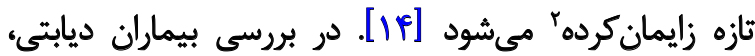

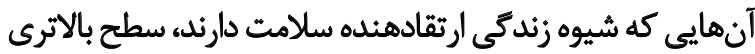

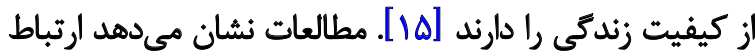

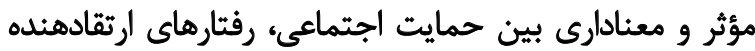

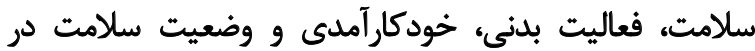

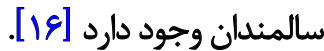

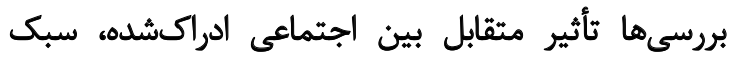

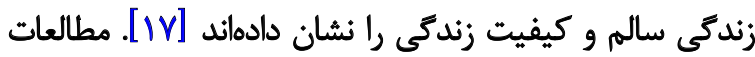

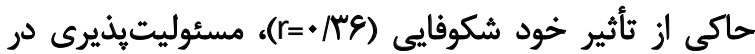

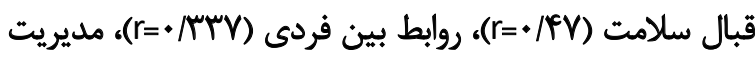

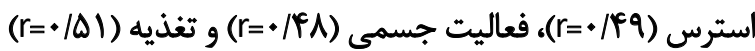

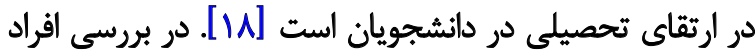

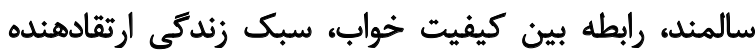

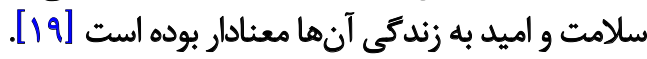

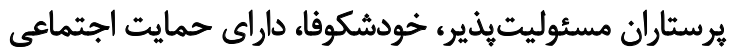

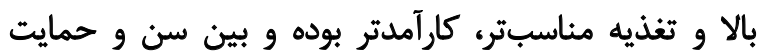

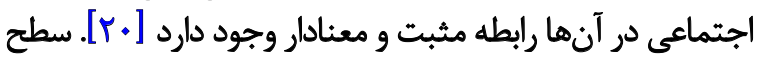

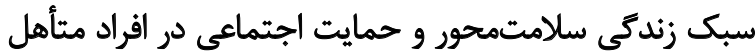

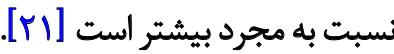

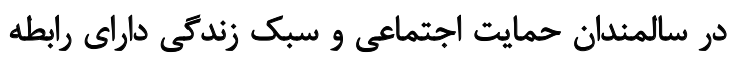

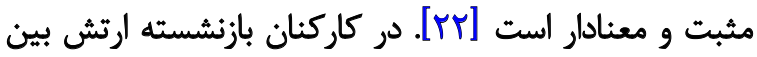

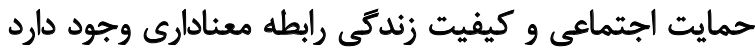

1. Health Promoting Life Style Profile

2. Postpartum 


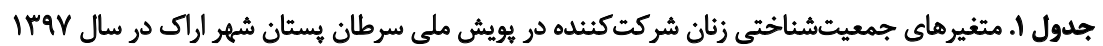

\begin{tabular}{|c|c|c|}
\hline تعداد (درصد) & \multicolumn{2}{|c|}{ متغير } \\
\hline$W E(W / e)$ & متأهل & \\
\hline$M(W N)$ & مجرد & وضعيث تأهل \\
\hline 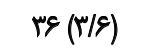 & مطلقه / بيوه & \\
\hline$W^{e}\left(v^{e} / f\right)$ & خانهدار & \\
\hline$r \Delta E(T \Delta / S)$ & كارمند & وضعيت شغلى \\
\hline$\Lambda \Delta \Delta(\Lambda \Delta / \Delta)$ & شهر & \\
\hline$f \cdot\left(f^{e}\right)$ & روسيتا & هحل سكونت \\
\hline $1.0(1.10)$ & حومه & \\
\hline$r q(V / q)$ & نسارد & \\
\hline$M T(M T / T)$ & خدمات درمائي & \\
\hline $810(8 / / 0)$ & تأمين اجتماعى & نوع بيمه \\
\hline $\operatorname{IV}(\mathrm{M} / \mathrm{)}$ & ارتش & \\
\hline$\Delta V(\Delta / N)$ & ديكر & \\
\hline $\operatorname{erq}(g r / 1)$ & نلناشته & \\
\hline mEN (re/9) & داثتله & وضعيت مهاجرت \\
\hline $\operatorname{lf\lambda }(1 F / 9)$ & خيلى ناكافى & \\
\hline MI9 (rT/I) & ناكافى & \\
\hline$F 1 \cdot(F / / F)$ & تا حدى كافى & سطح درآمد \\
\hline $199(r \cdot 11)$ & كافي & \\
\hline If $(1 / f)$ & خيلى كافي & \\
\hline $8 q(8 / 9)$ & خيلي بايين & \\
\hline$\pi(\pi)$ & بايين & \\
\hline era $(g / / \Delta)$ & متوسط & وضع اقتصادى \\
\hline$(\Delta / 9)$ & بالا & \\
\hline$V(\cdot / V)$ & خيلي بالا & \\
\hline$\Delta \cdot \Delta(\Delta Y / Y)$ & طبيعى & \\
\hline MTA (MPR) & اضاقه وزن & \\
\hline $1.0(1.19)$ & جات & نمايه توده بلديى (طبقهبندى ششده) \\
\hline$M(T / M)$ & خيلى هات & \\
\hline$\Delta E q(\Delta \& / q)$ & ندارد & \\
\hline 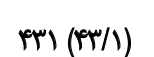 & دارد & اتوان تامين عُذا \\
\hline
\end{tabular}




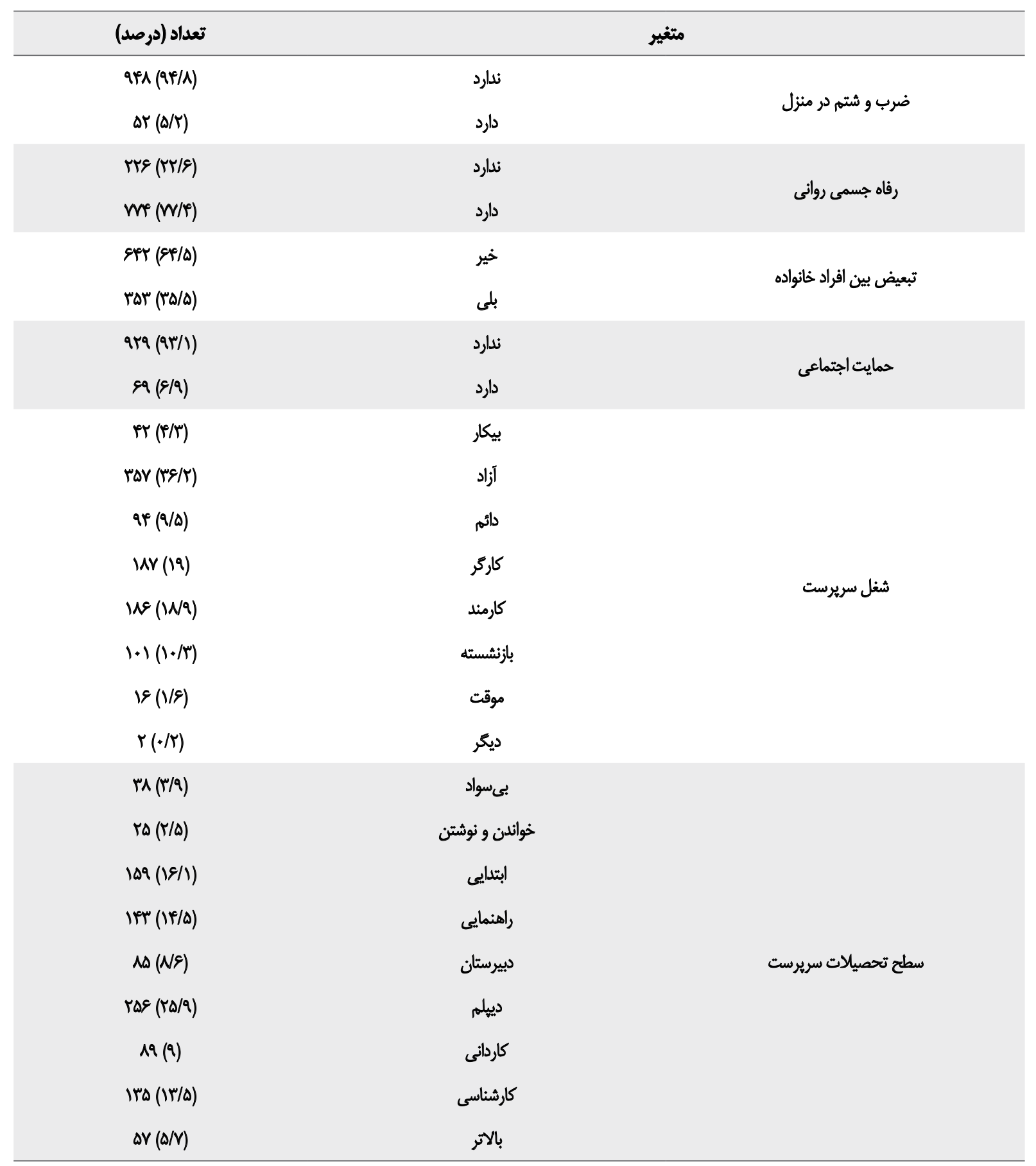

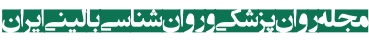

ترجمه و اصلاحشده فارسى سبك زندكى ارتقادهنده سلامت كه آن

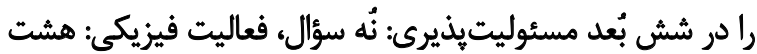

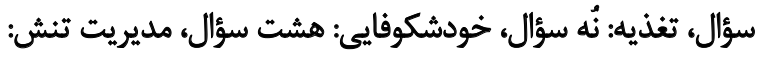

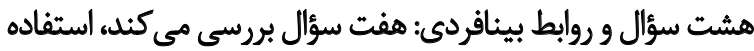

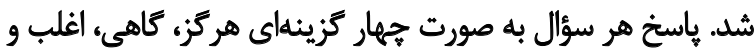

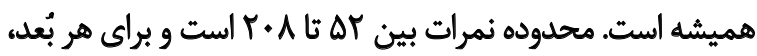

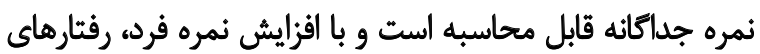
ارتقادهنده سلامت در سطح مطلوبترى قرار مى مئيرد.

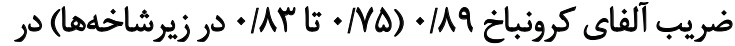

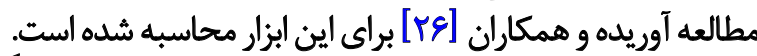

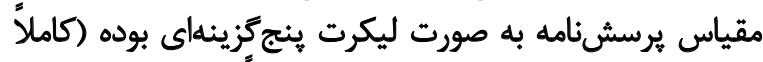

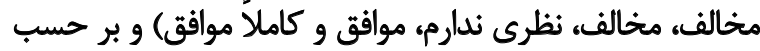

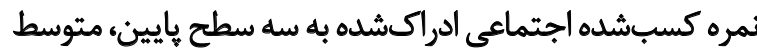
و بالا تقسيم مى شكود.

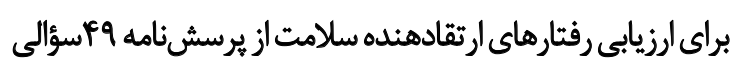


جدول r. رابطه سبكى زئدكى ارتقادهئده سلامت با اجتماعى ادراكشده در زنان شركت كنئده در يويش ملى سرطان يستان شهر اراك در سال IrqY

\begin{tabular}{|c|c|c|c|c|c|c|}
\hline \multicolumn{2}{|c|}{ ارتباط با سبك زندكى ارتقادهنده سلامت } & \multicolumn{2}{|c|}{ ارتباط با اجتماعى ادراكشده } & \multirow{2}{*}{ ميانغين土انحراف معيار } & \multirow{2}{*}{ تعداد } & \multirow{2}{*}{ مثغير } \\
\hline $\mathbf{p}$ & $\mathbf{r}$ & $\mathbf{p}$ & $\mathbf{r}$ & & & \\
\hline$\%$ & 1 & $\%$ &.$/ 4 T 9$ & 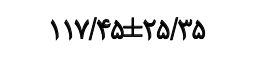 & $M r$ & سبك زندكى ارتثادهنده سلامث \\
\hline.$\cdots$ & ./erq & .1. & 1 & Pr/AYINAY & $1 \ldots$ & اجتماعي ادراكشده \\
\hline.$\ldots$ & $\cdot / F+\Delta$ & 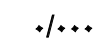 & . /AAS & $|g| \cdot V \pm T / T r$ & qua & حمايت خانوادكى \\
\hline.$\cdots$ &.$/ \mu \cdot$ &.$\cdots$ & - /ATY & IH/EN土M/EV & qua & حمايت دوستان \\
\hline$\%$ &.$/ r v e$ & $\%$ & - AART & $|Q| \cdot \& \pm m / M f$ & qu & حمايت سايرين مهمم \\
\hline$\%$ & - NAF & 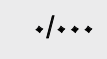 & - Trea & $\mid \varphi / \% \vee \pm \varphi / q q$ & $9 \vee r$ & فعاليث بلنىى \\
\hline$\%$ & . Mar & $\%$ &.$/ r q 4$ & $\mid V / \pi / \pm+4 / .$. & $9 \vee$. & وضعيت تغذيه \\
\hline$\%$ & $\cdot / 9 \cdot \Delta$ & $+\cdots$ &.$/ N \Delta \Delta$ & $M I / N A V / M F$ & 9re & مسئوليتيذيرى سلامت \\
\hline$\%$ &.$/ 1999$ & $+\cdots$ &.$/ f a r$ & $r F / \Delta V \pm \& / T \lambda$ & $1+\ldots$ & خودشكوفايى \\
\hline$\%$ & - /AIA & $\%$ & $\cdot / r A I$ & $1 / / A V \pm r / Q \Lambda$ & qs & كثترل استرس \\
\hline$\%$ & $\cdot / A \Delta V$ & $\%$ &.$/ F i 1$ & $|V / V \cdot \pm T / m|$ & Qr & ارتباط بينافردى \\
\hline.$\cdots$ &.$- / 1 r e$ & $.1 . .1$ & -.111 & $M / V A \pm 11 / T$. & qu & سن (سال) \\
\hline.$\cdots$ &.$/ M T$ & .1. &.$/ 19 Y$ & $11 / m 1 \pm f / 19$ & qAS & سطح تحصيلات (رال) \\
\hline.$/ . . r$ &.$/ 1 P f$ & $.1 .+r$ & . Mre & ETAIFT/A \pm PraIV./A & $\Delta r^{r}$ & درأمد سرائه (ريال) \\
\hline$. / 4+9$ & $-+1+40$ & . & $-+1+\pi r$ & $r \Delta / r I \pm r / \Psi \wedge$ & $9 \Delta V$ & نمايه توده بدنى (قد"/ وزن) \\
\hline
\end{tabular}

ع

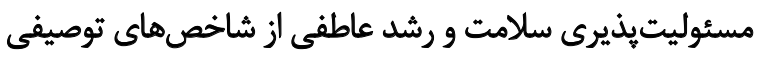

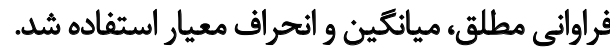

براي طبقهبندى BMI از روش استاندارد سازمان جهاني

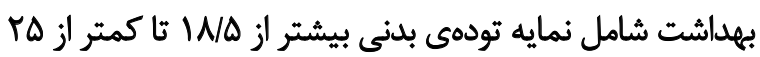

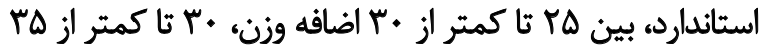

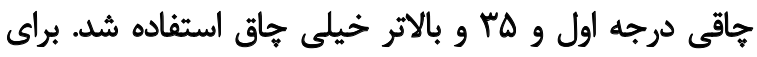

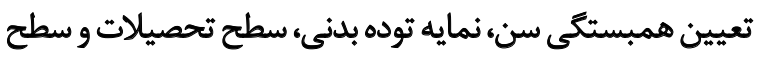

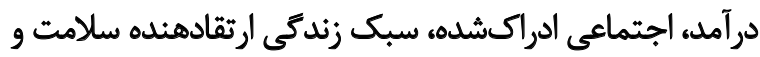

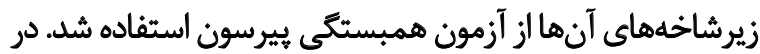

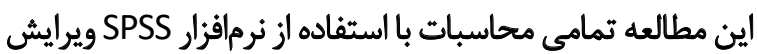

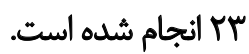

1. d.

از بين هزار نفر زنان شركت كنيده در يويش ملي سرطان يستان، فر صد مثأهل و VVIF

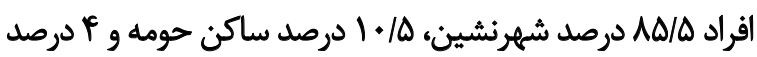

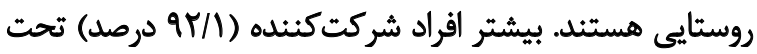

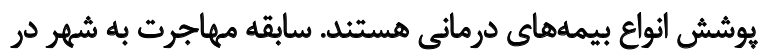

اين يرسشنامه توسط والكر و همكاران در سال 19V^ ارائه

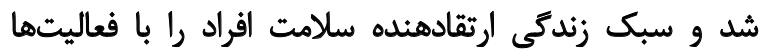

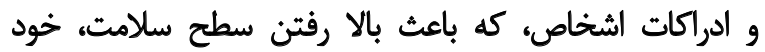

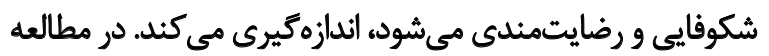

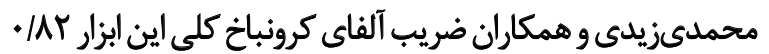

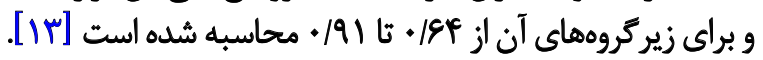
براى توصيف متغيرهاى روانى اجتماعى، مائند وضعيث تأهل،

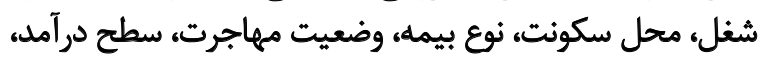

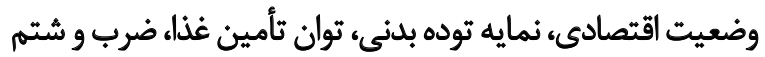

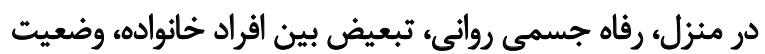

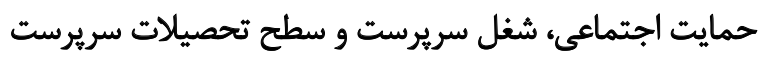

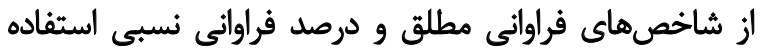

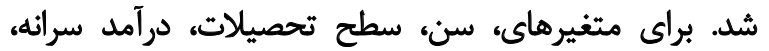

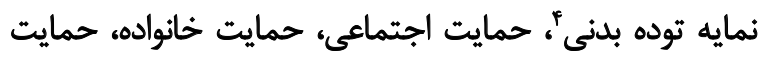

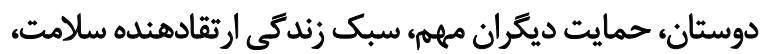

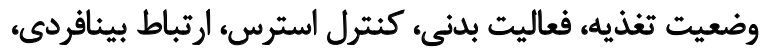

4. Body Mass Index 
(r=॰/r•)

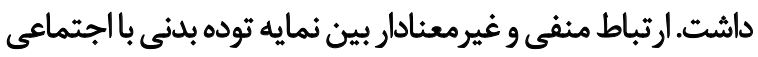

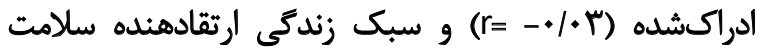

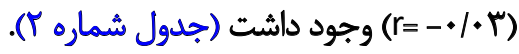

بحث

اين مطالعه با هدف بررسى ارتباط اجتماعى ادراكشده

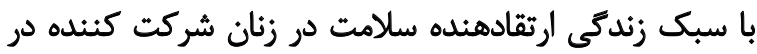

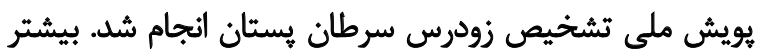

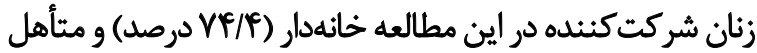

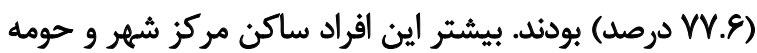

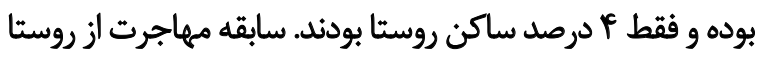

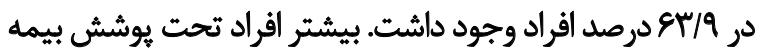

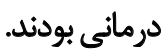
در مطالعه همبستكى مثبت، قابل قبول و مادئ معنادارى بين

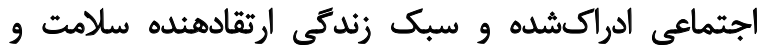

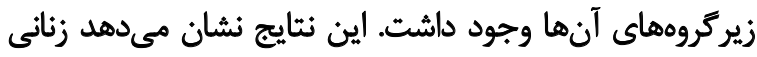

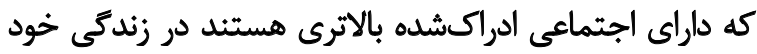

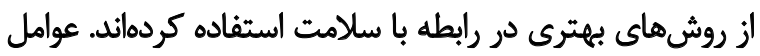

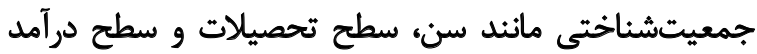

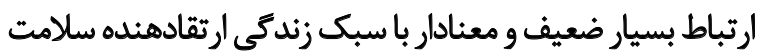
و اجتماعى ادراكشده داشتند.

مطالعه انجامشده توسط عليرضا كلدى و همكاران نيز نشان

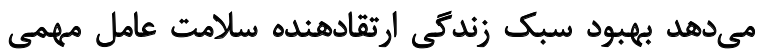

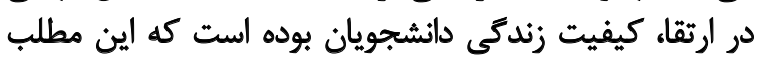

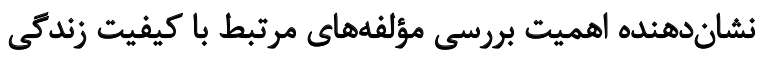

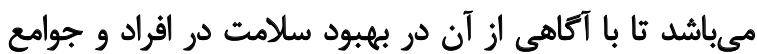

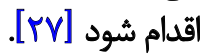

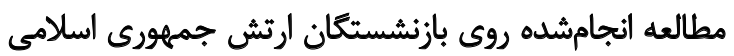

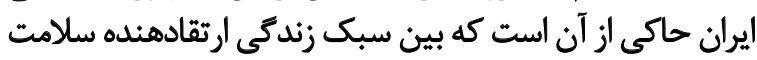

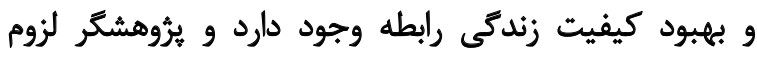

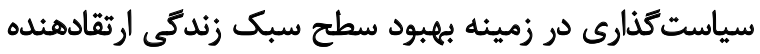

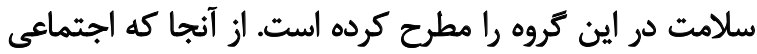

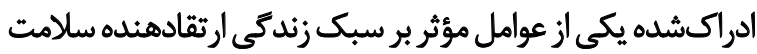

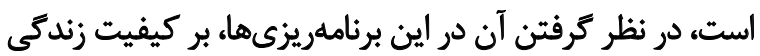

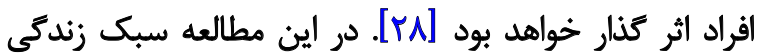

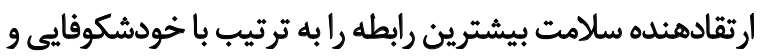

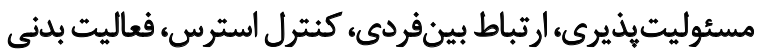

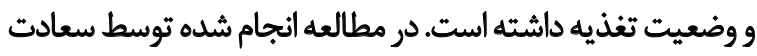

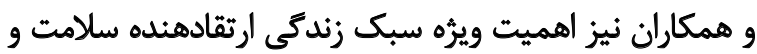

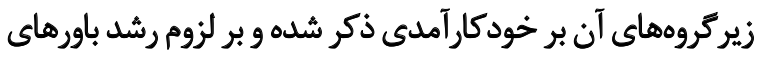

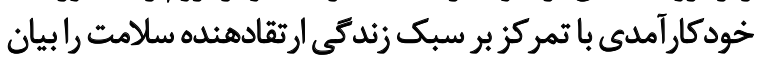

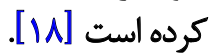

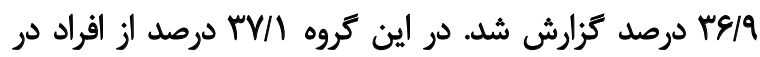

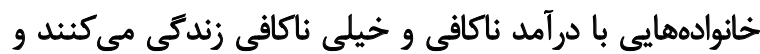

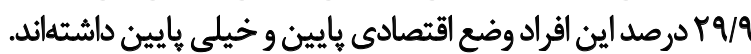

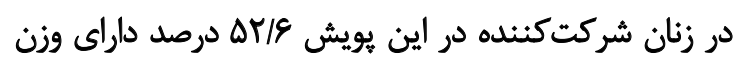

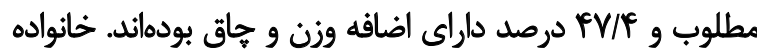

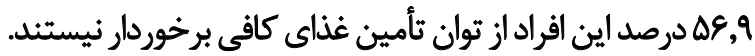

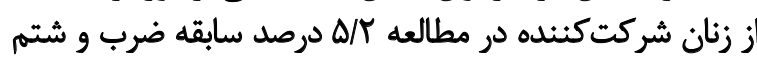

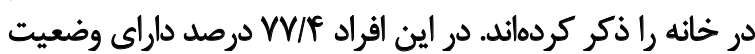

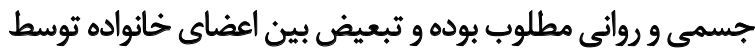

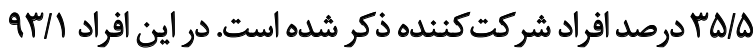

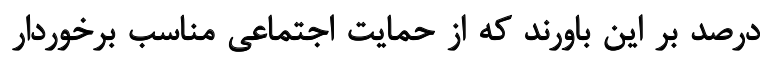

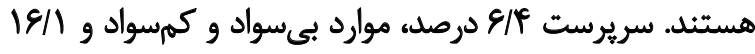

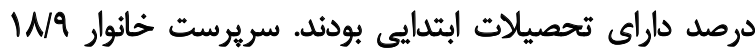

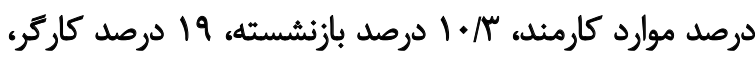

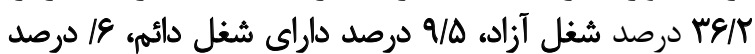

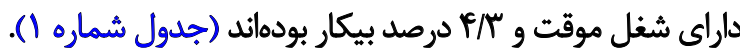

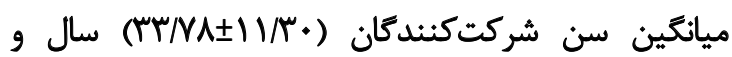

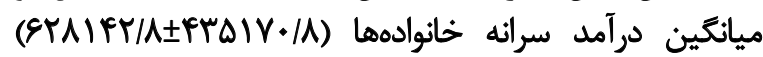

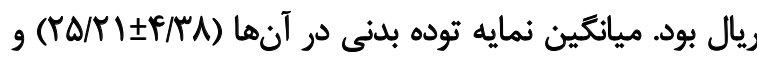

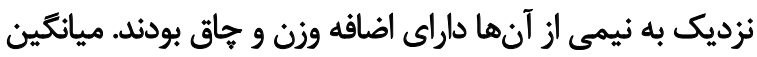

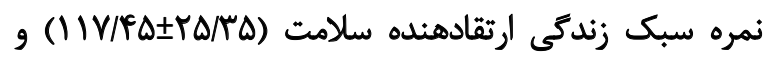

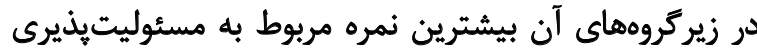

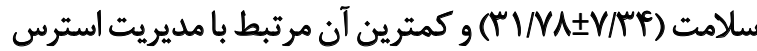

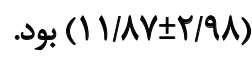

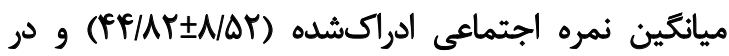

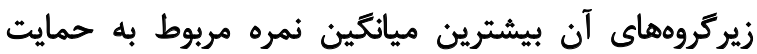

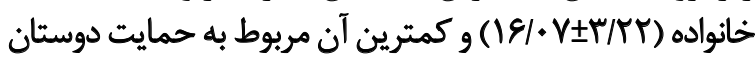

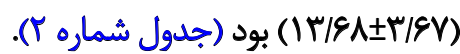

بيشترين همبستكى معنادار حمايت اجتماعي در ارتباط با بائ

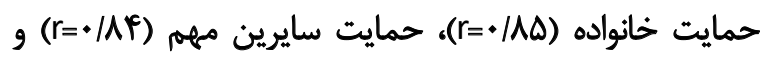

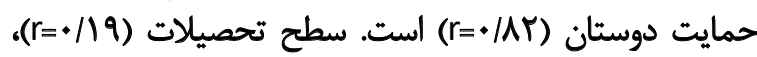

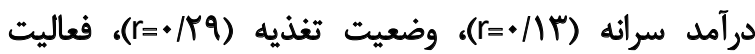

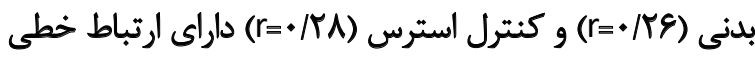

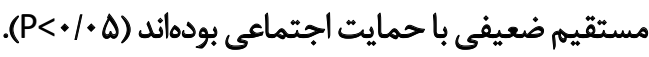

سبك زندگى ارتقادهنده سلامت رابطه قوى و معنادار با

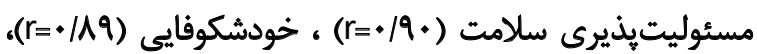

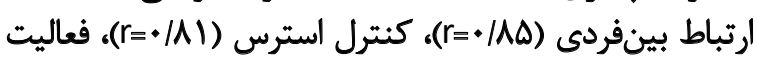

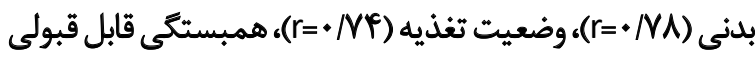

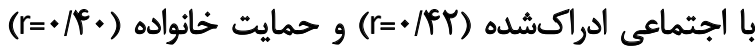

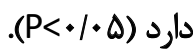

همبستَى پايين و معنادارى در رابطه با حمايث دوستان 


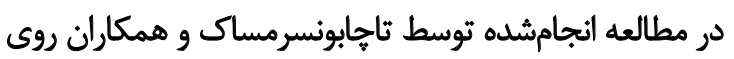

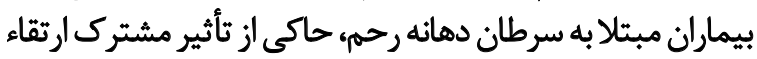

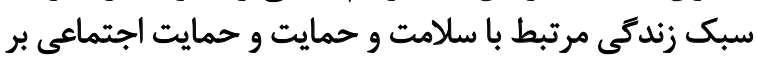

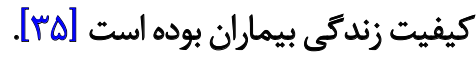

مطالعه انجامشده توسط آبيى و همكاران روى زوجين نابارور

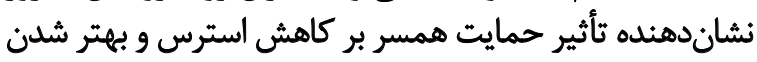

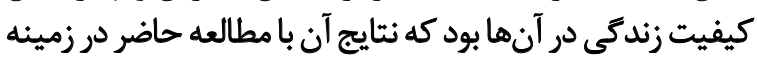

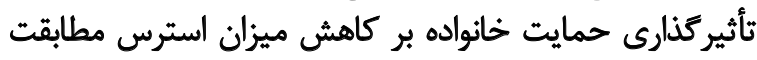

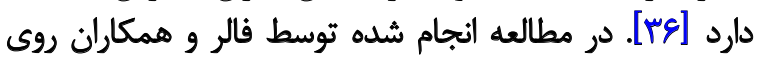

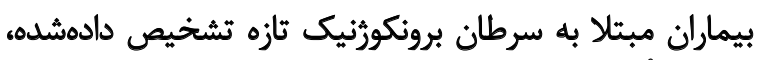

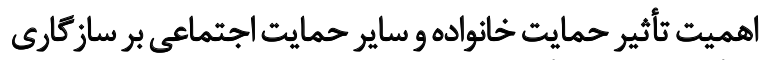
و تأثير درمان را تأييد كرده است [rV]

\section{نتيجلمكيرى}

طبق نتايج حاصل از مطالعه رابطه مستقيم و معنادارى بين إداعين

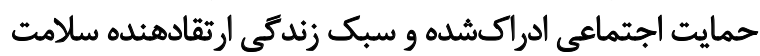

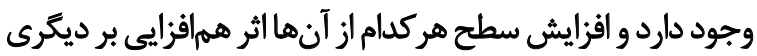

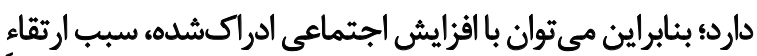

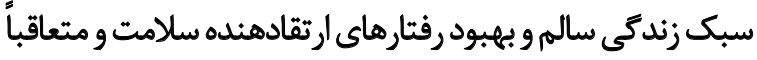

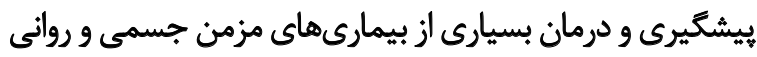

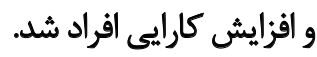

از جمله محدوديتهاي مطالعه حاضر اين بود كه با توجه

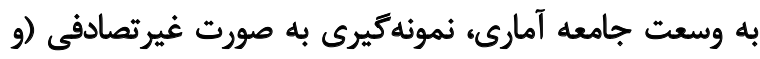

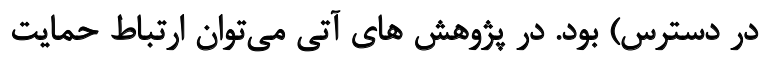

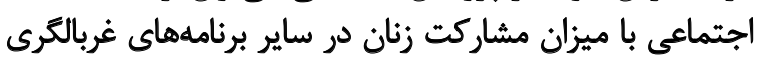

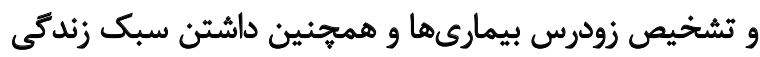

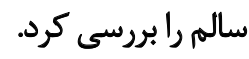

ماحظات اخلاقى

\section{يبيروى از اصول الخالاق يؤوهش}

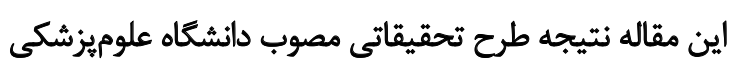

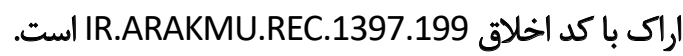

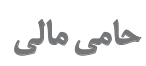

معاونت تحقيقات و فناورى دانشعاه علوميزشكى اراى حامى مالى اين يُروهش بوده است.

$$
\text { مشاركت ثويسند مكان }
$$

مفهومسازى: همه نويسندكان؛ تحقيق و بررسى، جمعآورى

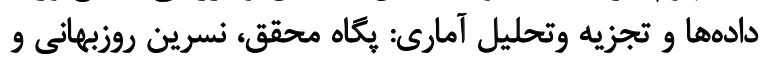

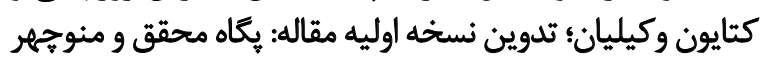

بررسى مطالعات نشان ميدهد سطح بالاتر سبك زندكى زئى

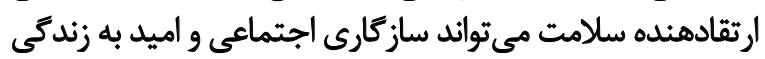

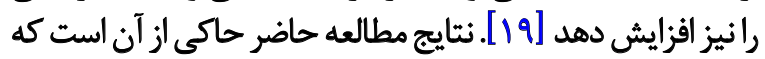

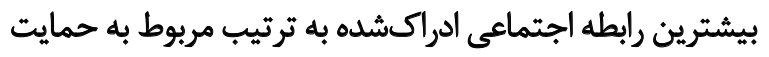

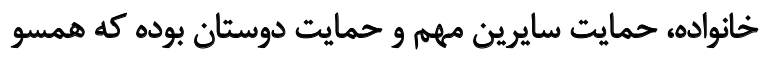

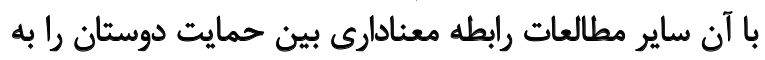

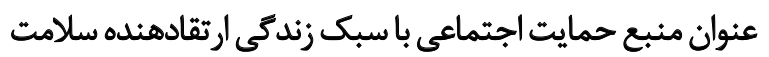

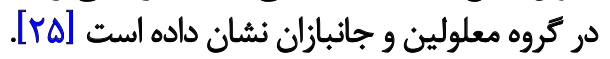

نتيجه مطالعه نشان مىدهد همبستكى معنادارى بين

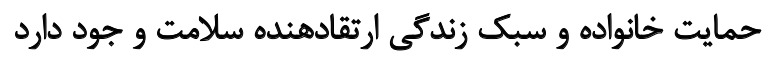

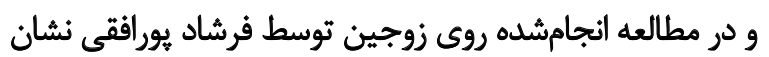

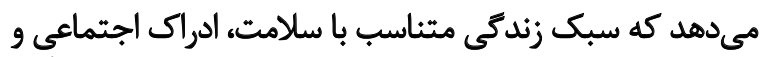

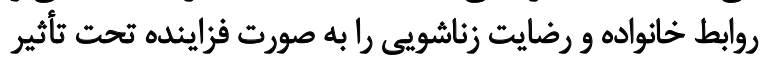
قرار مي دهد [rq].

نتيجه مطالعه حاكى از آن است كه رابطه مثبت و معنادارى بين

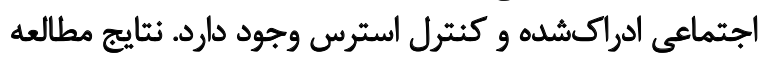

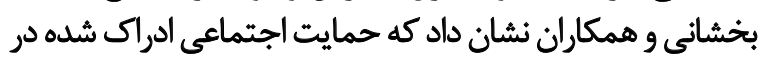

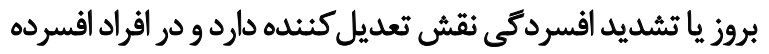

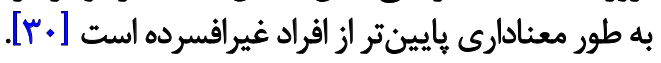
مطالعه انجامشده ثوسط شعبانزاده و همكاران نيز نشان باني

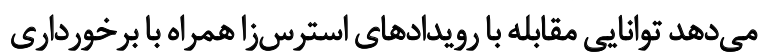

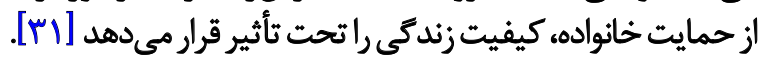

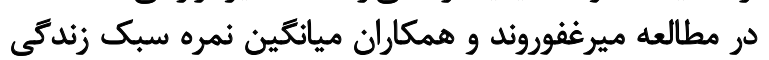

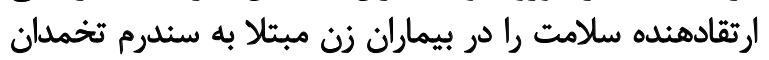

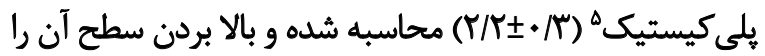

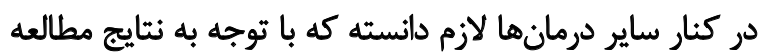

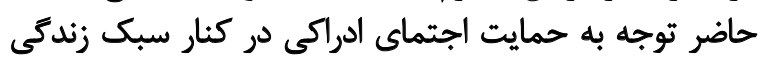

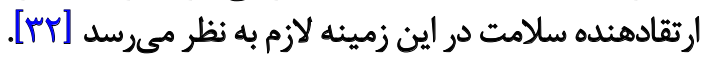

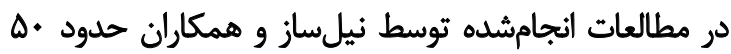

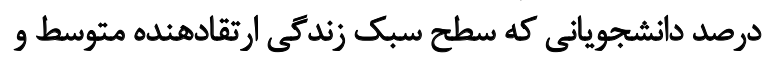

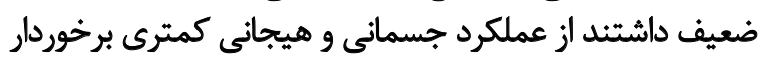

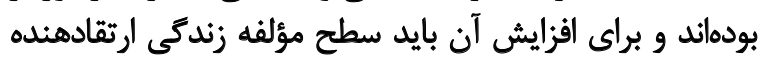

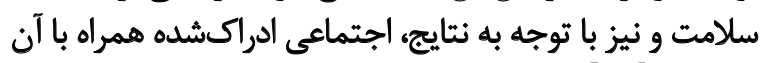
لارتقا يابد [سب]

بررسى انجام شده توسط رنكرونى و همكاران نشان مي دهد

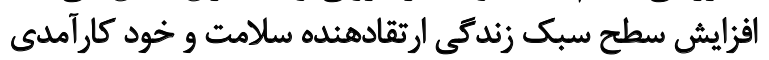

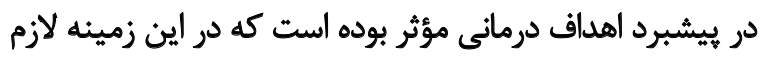

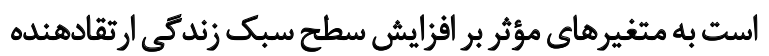

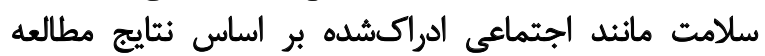

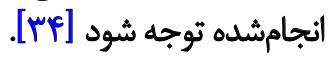


راديور؛ ويراستارى و نهايىسازى نوشته: يُكاه محقق.

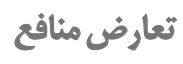

مطالعه حاضر تضاد منافعى با فرد يا سازمانى ندارد.

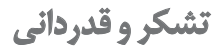

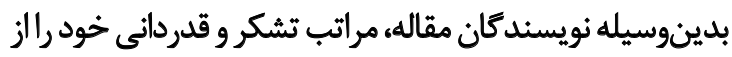

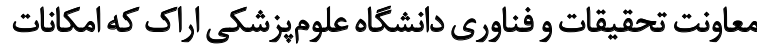

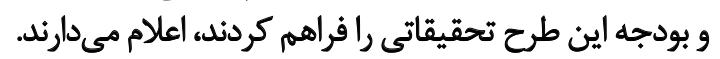




\section{References}

[1] Abdolkarimy M, Zareipour MHM, Dashti S, Faryabi R, Movahed E. [Health promoting behaviors and their relationship with self-efficacy of health workers (Persian)]. Iran Journal of Nursing. 2017; 30(105):68-79. [DOI:10.29252/ ijn.30.105.68]

[2] Noury Ghasemabady R, Bayanzadeh SA. [Social support and social network characteristics of chronic mental patients (Persian)]. Iranian Journal of Psychiatry and Clinical Psychology. 1996; 2(4):38-43. http:/ /ijpcp.iums.ac.ir/article1-1747-fa.html

[3] Chenary R, Noroozi A, Tahmasebi R. [Effective factors of health promotion behaviors based on Health Promotion Model in chemical veterans of Ilam province in 2012-13 (Persian)]. Scientific Journal of Ilam University of Medical Sciences. 2013; 21(6):257-67. http://sjimu.medilam.ac.ir/ article-1-911-fa.html

[4] Amarloo P, Shareh H. [Social support, responsibility, and organizational procrastination: A mediator role for basic psychological needs satisfaction (Persian)]. Iranian Journal of Psychiatry and Clinical Psychology. 2018; 24(2):176-89. [DOI:10.32598/ijpcp.24.2.176]

[5] Rezaeipandari H, Morowatisharifabad M. [Assessment of Psychosocial Determinants (Self-Efficacy and Social Support) of Lifestyle in the Elderly in Yazd City, Iran (Persian)]. Qom University of Medical Sciences Journal. 2016; 10(9):5160. http://journal.muq.ac.ir/article-1-502-en.html

[6] Amarloo P, Shareh H. Social [Support, Responsibility, and Organizational Procrastination: A Mediator Role for Basic Psychological Needs Satisfaction (Persian)]. Iranian Journal of Psychiatry and Clinical Psychology. 2018; 24(2):176-189. http://ijpcp.iums.ac.ir/article-1-2723-en.html

[7] Dai Y, Zhang C, Zhang B, Li Z, Jiang C, Huang H. Social support and the self-rated health of older people: A comparative study in Tainan Taiwan and Fuzhou Fujian province. Medicine. 2016; 95(24). [DOI:10.1097/MD.0000000000003881] [PMID] [PMCID]

[8] Javadifar N, Larki A, Javadnoori M, Haghighizadeh M. The relationship between perceived social support and lifestyle in middle-aged females of Ahvaz, Iran. Jundishapur Journal of Chronic Disease Care. 2016; 6. [DOI:10.17795/jjcdc-37111]

[9] Maryam Mataji Amirrood M, Taghdisi MH, Shidfar F, Mahmood Reza G. [The relationship between perceived social support and obesity preventive eating behavior in women of Urmia City in 2012 (Persian)]. Razi Journal of Medical Sciences. 2014; 21(119):1-11. http://rjms.iums.ac.ir/article1-3095-en.html

[10] Schiotz M, Bogelund M, Almdal T, Jensen B, Willaing I. Social support and self-management behaviour among patients with type 2 diabetes. Diabetic medicine: A Journal of the British Diabetic Association. 2012; 29(5):8. [DOI:10.1111/ j.1464-5491.2011.03485.x] [PMID]

[11] Shayeghian Z, Amiri PE, Aguilar-Vafaie M, Besharat M, Parvin M, Roohi Gilani K. The relationship between perceived social support and glycated hemoglobin in patients with type 2 diabetes: the moderating role of alexithymia. Iranian Journal of Diabetes and Lipid Disorders. 2015; 14(4):247-54. http://ijdld.tums.ac.ir/article-1-5399-en.html
[12] Fakhral-Sadat N, Shubo AM. Explaining the relationship between perceived social support and quality of life with the mediated role of perceived stress in female head of household in Sanandaj. Journal of Applied Sociology. 2017; 4(64)-99-116. https:/ / www.sid.ir/en/journal/ViewPaper.aspx?id=576169

[13] Mohammadi Zeidi I, Pakpour Hajiagha A, Mohammadi Zeidi B. Reliability and validity of Persian version of the health-promoting lifestyle profile. Journal of Mazandaran University of Medical Sciences. 2012; 21(1):102-13. http://jmums. mazums.ac.ir/article-1-955-fa.pdf

[14] Hajimiri K, Shakibazadeh E, Mehrizi AAH, Shabbidar S, Sadeghi R. The impact of general health and social support on health promoting lifestyle in the first year postpartum: The structural equation modelling. Electronic Physician. 2018; 10(1):6231-9. [DOI:10.19082/6231] [PMID] [PMCID]

[15] Ledinski Fičko S, Smrekar M, Hošnjak AM, Kurtović B, Kovačević I, Konjevoda V. Health promotion behaviors and quality of life among cancer patients-a systematic review. Journal of Applied Health Sciences=Časopis za Primijenjene Zdravstvene Znanosti. 2019; 5(1):87-96. https://doi. org/10.24141/1/5/1/8

[16] Fan W, Yu S. Social support network, social support, selfefficacy, health-promoting behavior and healthy aging among older adults: A pathway analysis. Archives of Gerontology and Geriatrics. 2019; 85:103934. https://www.sciencedirect. com/science/article/abs/pii/S0167494319301773

[17] Kalankash SP, Nemati S. Modeling structural relations of social support and identity styles with social adjustment and quality of life. Social Psychology Research. 2019; 8(32):79-90. http://www.socialpsychology.ir/article_89714.html?lang=en

[18] Sadat S, Kalantari M, Kajbaf MB. [The relationship between health promoting lifestyle components and academic selfefficacy in dormitory students (Persian)]. Research in Medical Education. 2017; 9(4):30-8. [DOI:10.29252/rme.9.4.38]

[19] Homaei R, Pooyanmehr M. [The relationship between health promoting life styles and sleep quality with social adjustment and life expectancy among elderly (Persian)]. Journal of Aging Psychology. 2018; 3(4):271-80. https:// doaj.org/article/91dae a729e514e829e053642c437df69?

[20] Mohammadbeigi A, Anbari Z, Mohammadsalehi N, Mahdipour A, Ahmadli R, Ansari H. [Study of the relationship between self-efficacy and health-promoting lifestyle and associated factors in nurses (Persian)]. Qom University of Medical Sciences. 2016; 10(7):49-57. http://journal.muq.ac.ir/article1-433-en.html

[21] Parsamehr M, Rasoolinejad SP. [Investigating the relationship between healthy lifestyle and social health among talesh city people (Persian)]. Social Development. 1394; 37(10):3566. https://qjsd.scu.ac.ir/article_11936_a8fbaee3bd08c656487624c6299935d6.pdf

[22] Rafiee S, Toozandehjani H, Ahooei MR. [Relationship of lifestyle and social support with marital satisfaction of elderly population (Persian)]. Salmand: Iranian Journal of Ageing. 2016; 11(2):226-33 [DOI:10.21859/sija-1102226]

[23] Goudarz T, Vahdani nia V. [The relationship between "Quality of Life" and social support of the retired military personnel (Persian)]. Military Caring Sciences. 2019; 5(4):263-72. [DOI:10.29252/mcs.5.4.263] 
[24] Mohammad-Alizadeh-Charandabi S, Mirghafourvand M, Tavananezhad N, Karkhaneh M. [Health promoting lifestyles and self-efficacy in adolescent boys (Persian)]. Journal of Mazandaran University of Medical Sciences. 2014; 23(109):15262. http://jmums.mazums.ac.ir/article-1-3386-en.html

[25] Chenary R, Noroozi A, Noroozi R. [The relationship between perceived social support and health promoting behaviors in chemical veterans of Ilam Province (Persian)]. Iranian Journal of War and Public Health. 2013; 21(6):1-10. http://ijwph.ir/article1-328-en.html

[26] Avarideh S, Majareh SA, Moghtader L, Abedini M, Bozorgi AM. The Mediating role of perceived social support in the effect of interpersonal forgiveness on social health in students. Knowledge \& Research in Applied Psychology. 2019; 20(1):71-80. https://www.sid.ir/en/journal/ViewPaper.aspx?id=668911

[27] Kaldi A, Kabiran EH, Mohagheghi KS, Rezasoltani P. [The evaluation of relationship between health-promoting lifestyle and quality of life (case of study: University of Social Welfare And Rehabilitation Sciences Students in Tehran) (Persian)]. Journal of Iranian Social Development Studies. 2014; 6(4):87-95. http://jisds. srbiau.ac.ir/article_6804.html

[28] Goodarz Tele Jardi M, Leh Vahdani Nia V. [The relationship between quality of life and social support in retirement community army of the Islamic Republic of Iran (Persian)]. Journal of Army Nursing Faculty of the Islamic Republic of Iran. 2019; 5(4):263-72. http:/ / mcs.ajaums.ac.ir/article-1-247-fa.html

[29] Pourofoghi F. [The role of lifestyles, social perception and family relationships in predicting of marital satisfaction the young teachers (Persian)]. Journal of School Psychology. 2016; 5(2):20-38. http://jsp.uma.ac.ir/article_438_2e08e144469cd566ff8 0a98c81595200.pdf?lang=en

[30] Bakhshani NM, Birashk B, Atefvahid MK, Bolhari J. [Correlation of social support and negative life events with depression (Persian)]. Iranian Journal of Psychiatry and Clinical Psychology. 2003; 9(2):49-55. http://ijpcp.iums.ac.ir/article-1-190-en.html

[31] Shabanzadeh A, Zare Bahramabadi M, Hatami HR, Zahrakar K. [Investigating the relationship between coping styles and quality social support The life of women heads of households in Tehran (Persian)]. Journal of Women and Society. 2013; 4(4):1-20. http:/ / ensani.ir/file/download/article/20151011145423-10001-23.pdf

[32] Mirghafourvand M, Mohammad-Alizadeh Charandabi S, Behroozi lak T, Aliasghari F. [Assessment of health promoting lifestyle status and its socio-demographic predictors in women with Polycystic Ovarian Syndrome (Persian)]. Journal of Faculty of Nursing and Midwifery, Tehran University of Medical Sciences (HAYAT). 2016; 22(4):394-407. http://hayat.tums.ac.ir/ article-1-1662-en.html

[33] Nilsaz M, Tavassoli E, Mazaheri M, Sohrabi F, Khezli M, Ghazanfari Z, et al. [Study of healthpromoting behaviors and life style among students of Dezful universities (Persian)]. Journal of Ilam University of Medical Sciences. 2013; 20(5):168-75. http://sjimu. medilam.ac.ir/article-1-940-en.html

[34] Roncoroni J, Tucker CM, Wall W, Wippold G, Ratchford J. Associations of health self-efficacy with engagement in healthpromoting behaviors and treatment adherence in rural patients. Family \& Community Health. 2019; 42(2):109-16. [DOI:10.1097/ FCH.0000000000000219] [PMID]

[35] Taechaboonsermsak P, Kaewkungwal J, Singhasivanon P, Fungladda W, Wilailak S. [Causal relationship between health promoting behavior and quality of life in cervical cancer patients undergoing radiotherapy (Persian)]. Southeast Asian Journal of Tropical Medicine and Public Health. 2005; 36(6):1568-75. https://www.tm.mahidol.ac.th/seameo/2005_36_6/29-3673.pdf

[36] Abbey A, Andrews FM, Halman LJ. Provision and receipt of social support and disregard: What is their impact on the marital life quality of infertile and fertile couples? Journal of Personality and Social Psychology. 1995; 68(3):455-69. [DOI:10.1037/00223514.68.3.455] [PMID]

[37] Faller H, Lang H, Schilling S, Wagner J. Coping with illness in bronchial cancer from the viewpoint of the patients, their relatives and caregivers. A five-level assessment. Psychotherapie, Psychosomatik, Medizinische Psychologie. 1992; 42(9-10):322-31. [PMID] 
This Page Intentionally Left Blank 